\title{
Erratum: Mixed quantum-classical dynamics of an amide-I vibrational excitation in a protein $\alpha$-helix [Phys. Rev. B 82, 174308 (2010)]
}

\author{
Holly Freedman, Paulo Martel, and Leonor Cruzeiro \\ (Received 28 July 2011; revised manuscript received 7 September 2011; published 7 October 2011)
}

DOI: 10.1103/PhysRevB.84.139902 PACS number(s): 82.20.Ln, 82.20.Wt, 87.15.bd, 87.10.Hk, 99.10.Cd

In the GROMACS code modifications, instead of the nanometer unit for the distance that is standard in GROMACS, a unit of $1 \AA$ was previously assumed. This led to dipole-dipole interactions between amide I vibrations at different sites and the interaction energies of the amide I vibration with the protein hydrogen bonds being overestimated, respectively, by three orders and by one order of magnitude. In addition, the quantum mechanical force terms were overestimated because, through the same error, the sites defined as hydrogen-bonded in the protein were not properly identified. Because the influence of the quantum vibration on the conformation of the polypeptide is less pronounced when these errors are corrected, the simulation times are increased to $10 \mathrm{ps}$. Moreover, each of the 20 simulation runs, obtained by varying the seed of the random number generator for the initial velocities, was repeated ten times in order to sample different quantum Monte Carlo paths and to yield better statistical estimates. Thus, the results presented here, for each value of $\chi$, represent averages over 200 simulation trajectories.

Two other changes are made in the current modified GROMACS code. The first of these makes sure that the interaction energy term varies continuously with the length of the hydrogen bond, even when the hydrogen bond breaks. This term is calculated as

$$
\hat{H}_{\mathrm{int}}=\chi \sum_{n=1}^{N}\left[\left(d_{n}-d_{e q}\right) \hat{a}_{n}^{+} \hat{a}_{n}\right],
$$

where $\chi=d \varepsilon / d\left(\left|R_{n}^{O}-R_{m}^{N}\right|\right)$ is the parameter of nonlinearity and $d_{e q}$ denotes the equilibrium length of the hydrogen bond between the carbonyl oxygen atom at site $n$ at position $R_{n}^{O}$ and the amine nitrogen atom at site $m$ at position $R_{m}^{N} \cdot d_{e q}$ is set

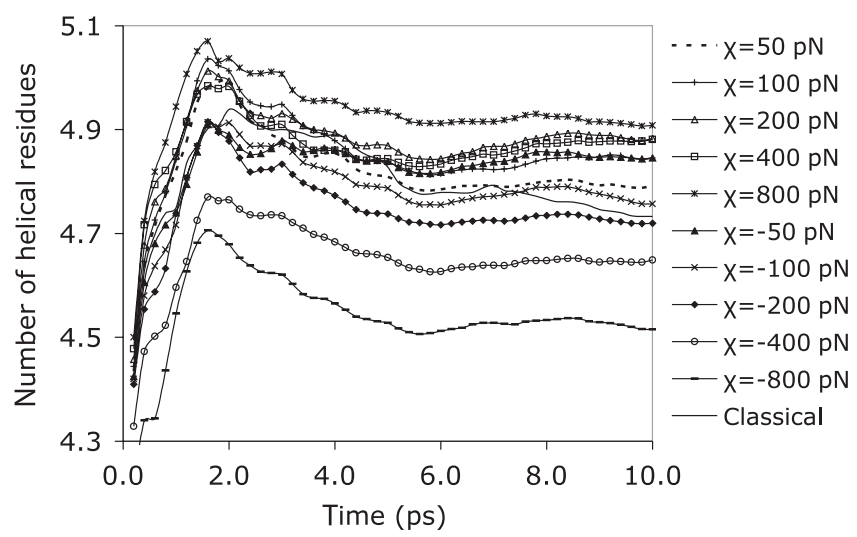

FIG. 1. Cumulative average of the number of helical residues as a function of simulation time (ps) determined by the g_helix GROMACS analysis tool.

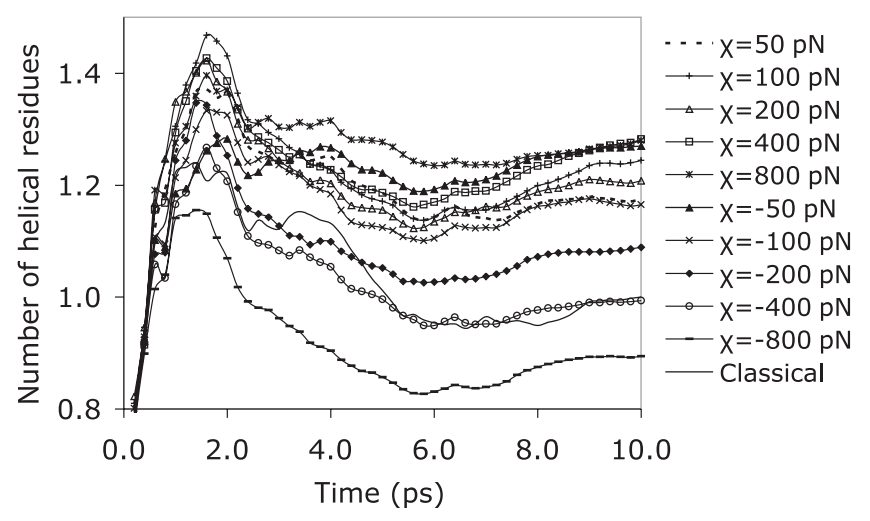

FIG. 2. Cumulative average of the number of helical residues as a function of simulation time (ps) determined by the do_dssp GROMACS analysis tool.

equal to $0.3 \mathrm{~nm}$ according to the force field. $d_{n}$ is the smallest hydrogen-bond distance between sites $n$ and $m$, as the sites $m$ are varied, assuming that a hydrogen bond only exists when $\left|R_{n}^{O}-R_{m}^{N}\right|<0.35 \mathrm{~nm}$ and $n>m+3$; if no such hydrogen bond exists, $d_{n}$ is set equal to $0.35 \mathrm{~nm}$.

Second, a slight modification is made in the Monte Carlo selection of the quantum vibrational eigenstate. We stipulate that the state into which the amide-I vibration is given a chance to propagate, according to a Metropolis-Monte Carlo step, should be most localized, i.e., should have the greatest probability amplitude, at a site different from the previous one. This is performed to allow for a greater chance of propagation of the quantum vibration to another site, at each time step.

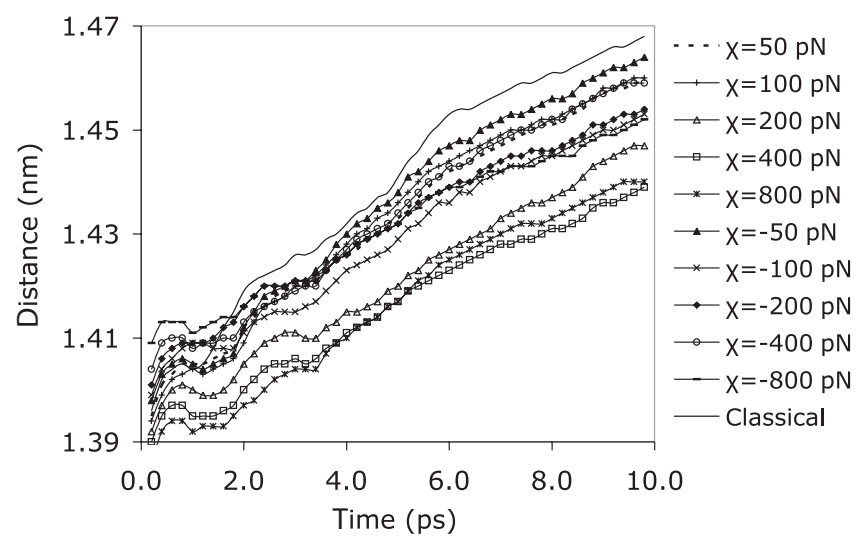

FIG. 3. Cumulative average of the minimum distance from the amino-terminal Ace group to the carboxy-terminal $\mathrm{NH}_{2}$ group as a function of simulation time (ps) determined by the g_mindist GROMACS analysis tool. 